\title{
Epidemic Threshold and Lifetime Distribution for Information Diffusion on Simultaneously Growing Networks
}

\author{
Emily M. Fischer \\ School of Operations Research \\ and Information Engineering \\ Cornell University \\ Ithaca, NY, USA \\ ef363@cornell.edu
}

\author{
Souvik Ghosh \\ LinkedIn \\ Sunnyvale, CA, USA \\ sghosh@linkedin.com
}

\author{
Gennady Samorodnitsky \\ School of Operations Research \\ and Information Engineering \\ Cornell University \\ Ithaca, NY, USA \\ gs18@cornell.edu
}

\begin{abstract}
We study information diffusion modeled by epidemic models on a class of growing preferential attachment networks. We show through a thorough simulation study that there is a fundamental difference in the nature of the epidemic process on growing temporal networks in comparison to the same process on static networks. The empirical distribution of the epidemic lifetime on growing networks has a considerably heavier, and possibly infinite, tail. Furthermore, the notion of the epidemic threshold has only minor significance in this context, since network growth reduces the critical value of the corresponding static graph.

Index Terms - information diffusion, epidemic models, tempo-
\end{abstract} ral networks, time-varying networks, preferential attachment

\section{INTRODUCTION}

Social networks and the world-wide web in general have a huge influence over our lives today. Many depend on the power of information diffusion on social networks - almost every company has marketing strategies for social networks, news agencies use social networks to increase their site traffic, and celebrities use social network to increase their brand value. Unfortunately, we also see harmful uses of this mechanism where adversaries spread fake news to drive their propaganda. Understanding the spread of information on networks is thus a topic of great interest, see [1], [6].

Epidemic models, which were historically developed to study disease spread in a network, have been applied to model information diffusion on the web, see [6], [13]. The study of epidemic spreading processes on complex networks in general has been a research topic of interest for a long time, typically under classical models like Susceptible-Infectious-Susceptible (SIS) and Susceptible-Infectious-Recovered (SIR) [9]. Recent Permission to make digital or hard copies of all or part of this work for personal or classroom use is granted without fee provided that copies are not made or distributed for profit or commercial advantage and that copies bear this notice and the full citation on the first page. Copyrights for components of this work owned by others than the author(s) must be honored. Abstracting with credit is permitted. To copy otherwise, or republish, to post on servers or to redistribute to lists, requires prior specific permission and/or a fee. Request permissions from permissions@acm.org

ASONAM '19, August 27-30, 2019, Vancouver, BC, Canada

(C) 2019 Copyright is held by the owner/author(s). Publication rights licensed to ACM.

ACM ISBN 978-1-4503-6868-1/19/08?/\$15.00

https://doi.org/10.1145/3341161.3342891 efforts have been made on time-varying or temporal networks, in which nodes and edges exist for only a subset of the entire time [3], [8], [12]. We consider an SIS model on a specific class of temporal networks: those that are strictly increasing in time.

In the epidemic process modeled after the SIS model, nodes exist in one of two possible states, Susceptible and Infected. A susceptible node may become infected at the next time step if it is adjacent to an infected node. An infected node transmits the infection to the susceptible node in the next time step with probability $\lambda$. The higher the value of $\lambda$, the higher the rate of transmission of the infection and the longer the infection persists in the network. A notable result specifies an epidemic threshold $\lambda_{c}$, below which the infection dies out quickly, and above which the infection may survive for a "long" time [4]. The notion of "long" depends on the cardinality of the node set, as the SIS process on an infinite graph may survive for an infinite amount of time, while a finite graph necessarily dies out in finite time.

Most theoretical results on epidemic threshold are limited to static graphs, that do not change over time. In the specific case of a growing complete graph, it has been proved that infinite survival is possible [11], however, to our knowledge this result has not been extended to more general graphs. We conduct a thorough simulation study of the SIS model on a class of graphs generated by the Preferential Attachment (PA) model. The PA model is a generative graph process where we start with a single node and zero edges and at each time step, we add a new node and an edge between this node and an existing node. The existing node for the new edge is selected with probability proportional to its degree. Note that while all graphs in the sequence are finite, the limiting graph is infinite. A major difference in our model from other work is that the epidemic process continues concurrently with the PA model.

We use simulations to compare the lifetime distributions of SIS processes on growing graphs versus finite static graphs. Our computational results show that the distribution of an infection's lifetime has a heavier tail in the growing case 


\section{IEEE/ACM International Conference on Advances in Social Networks Analysis and Mining}

compared to the static case. Contrary to the static case, the empirical lifetime distribution can be classified as long-tailed. Further, for large enough values of the spreading rate $\lambda$, we give evidence that infinite survival may be possible on the growing graph. We also argue that, unlike static graphs, the epidemic threshold for long survival does not depend on initial graph size.

The main contribution of our paper is that we show that the epidemic lifetime distribution for the SIS model on a growing network is significantly different from that in the case of a static network. Through simulations we find a strong evidence that

- there is a critical value $\lambda_{c}$ such that for $\lambda>\lambda_{c}$, the lifetime is infinite with a positive probability,

- lifetime distribution has a heavier tail compared to that on a static graph, and

- the typical notion of epidemic threshold is less pertinent in the case of growing networks.

\section{RELATED WORK}

\section{A. SIS Model}

A continuous-time SIS model on a finite graph $G$ is characterized by an infection rate $\nu>0$ and a recovery rate $\delta>0$. On a graph of $n$ nodes, we define the vector process $\left(X_{t}, t \geq 0\right), X_{t} \in\{0,1\}^{n}$, where we say that a node $i$ is infected at time $t$ if $X_{t}(i)=1$, and susceptible if $X_{t}(i)=0$.

An infected node becomes susceptible again after an exponentially distributed amount of time with rate $\delta$. For every edge $(i, j)$, there is a Poisson process with rate $\nu$ representing contact between nodes $i$ and $j$. If one node is susceptible and the other is infected at a contact time, then the susceptible node becomes infected. That is, if there is an arrival at time $t$, and $X_{t^{-}}(i)=1, X_{t^{-}}(j)=0$, then we set $X_{t}(j)=1$.

Without loss of generality, we can define the effective spreading rate $\beta=\nu / \delta$, and consider the recovery rate to be 1 . Thus the parameter space is truly only one-dimensional, in $\beta>0$ [10].

Often of interest is a critical $\beta_{c}$, with the property that if the spreading rate is greater than the critical value, then there is a positive probability that an infection will survive for a sufficiently long time. On an infinite graph $G_{\infty}$, a process with spreading rate $\beta>\beta_{c}$ has positive probability of infinite survival, and a process with spreading rate $\beta<\beta_{c}$ is guaranteed to die out in finite time. It has been stated that infinite scale-free networks have $\beta_{c}=0$ [2].

Infections on finite graphs necessarily have finite lifetime, so Ganesh et al. divide extinction time into two regimes. On a finite graph with $n$ nodes, extinction is said to be quick when $E[\tau]=O(\log n)$ and slow when $E[\tau]=\Omega\left(e^{n^{\alpha}}\right)$ for some $\alpha>0$. It has been established that a sufficient condition for quick extinction on finite graphs is $\beta<\frac{1}{\rho}$, where $\rho$ is the spectral radius of the graph [4]. Then there may exist a critical value $\beta_{c} \geq \frac{1}{\rho}$, above which the process may survive for a long amount of time. The value $\beta_{c}$ is often referred to as the epidemic threshold.
For finite graphs grown via preferential attachment, the critical value is shown to be inversely related to the size of the graph [4]. As a preferential attachment graph increases in size, the critical value decreases, with limit 0 as size increases to infinity.

\section{B. Time-varying networks}

Many real-life networks change over time and are called temporal or time-varying graphs [7]. For example, an email communication network may contain different connections during work hours than during evening hours. These types of networks, where edges disappear and reappear periodically, are discussed in [9], and a mean-field method has been developed for more general classes of time-varying networks in [5]. Some graphs like online social networks are constantly changing and are mostly growing over time.

The vast majority of epidemic research considers spreading processes on static graphs: the graph remains fixed as the virus propagates, and has no interaction with the spreading process. One interesting study on temporal graphs considers the interaction of SIR spreading on a preferential attachment network where nodes and edges arrive over time, but nodes are removed (along with their corresponding edges) after being infected [3]. Interestingly, the remaining nodes were seen to have a degree distribution that decreased from a power law to exponential as infection rate increased.

In this paper, we consider the class of preferential attachment graphs that are increasing with time and do not interact with the spreading process. Nodes and edges arrive independently according to the usual preferential attachment rules, and the infection may access new edges and nodes as soon as they are available. This model allows us to directly understand the effect of network growth on a SIS-type spreading process.

\section{MODEL}

We consider a discrete-time model with a graph sequence generated by the preferential attachment model. Letting $P A_{0}$ be the graph with a single node and zero edges, we generate recursively. Given $P A_{n}$, we let $D_{n}(w)$ denote the degree of node $w$ at time $n$, and we form $P A_{n+1}$ by

- adding a new node $v$, and

- forming the edges $\left(v, w_{i}\right), i=1, \ldots, m$, where $w_{i}$ is selected with probability

$$
\frac{D_{n}\left(w_{i}\right)}{m n+i-1} .
$$

We define the growing graph sequence $G=\left(G_{0}, G_{1}, G_{2}, \ldots\right)$ where $G_{0}$ is $P A_{n_{0}-1}$ and has $n_{0} \geq 1$ nodes and $G_{n}=$ $P A_{n+n_{0}-1}$ is a subgraph of $G_{n+1}$ for all $n \geq 0$.

A discrete-time SIS model on the graph sequence $G$ is characterized by the state vector $X_{n}$ and is defined as follows:

- Initialize $X_{0} \in\{0,1\}^{n_{0}}$.

- If $X_{n}(i)=1$ at time $n \geq 0$, then set $X_{n+1}(i)=0$. 
- If $X_{n}(j)=0$ or $j$ is born at step $n+1$, let $M$ be the number of neighbors $i$ of $j$ in $G_{n+1}$ such that $X_{n}(i)=1$,

$$
M:=\sum_{i} A_{n+1}(i, j) \mathbb{1}\left(X_{n}(i)=1\right),
$$

where $A_{n+1}$ is the adjacency matrix of $G_{n+1}$. Set

$$
X_{n+1}(j)= \begin{cases}1 & \text { with probability } 1-(1-\lambda)^{M} \\ 0 & \text { with probability }(1-\lambda)^{M}\end{cases}
$$

- The extinction time, or lifetime, of the process is

$$
\tau:=\inf \left\{n \geq 0: X_{n}(i)=0 \text { for all } i=1,2, \ldots, n_{0}+n\right\} .
$$

We assume that all infected nodes always recover in exactly 1 time step. The sole parameter in the model, $\lambda \in(0,1)$, is the transmission probability and is related to the transmission rate $\beta$ as

$$
\lambda=1-e^{-\beta} .
$$

\section{Simulation AND Results}

In our simulations, unless stated otherwise, we use $n_{0}=$ 1000, $m=1$ and let

$$
\begin{aligned}
G_{0} & =P A_{n_{0}-1} \\
G_{n} & =P A_{n_{0}-1+n}, \quad n=1,2,3, \ldots .
\end{aligned}
$$

Note that $G_{n}$ has $n_{0}+n$ nodes and $m\left(n_{0}+n-1\right)$ edges. The simulation is initialized by creating the graph sequence $G=\left(G_{0}, G_{1}, \ldots, G_{5000}\right)$ and randomly labeling half of the node population as infected, and the other half as susceptible at $n=0$. The discrete-time SIS model then runs until extinction or until timestep to $n=5000$ (with final graph size of $n_{0}+5000=6000$ ), whichever comes first. At least 1000 iid observations of termination time were recorded for the values of $\lambda=0.10,0.11, \ldots, 0.21$.

Network growth has the effect of prolonging the lifetime of an infection. The availability of new nodes and edges allows the infection to spread over a larger area, and when the infection dies out on the original portion of the graph, it may still be alive on the newer portion of the graph. This implies that the lifetime of an infection on the static graph $G_{0}$ is stochastically dominated by extinction time over $G=\left(G_{0}, G_{1}, \ldots\right)$. In this case, the static graph would give a lower bound on extinction time and probability of infinite survival, and an upper bound on the critical value for long survival.

We observe that a SIS process on a growing graph sequence has a fundamentally different lifetime distribution and a notion of critical value as compared to the SIS process on a static graph. Intuitively, the longer the process survives, the larger the graph, and hence the easier the spreading and the more likely it is to continue to survive. This contributes to a heavier-tailed lifetime distribution and the possibility of infinite survival. Further, it diminishes the notion of a critical spreading rate, because critical rates tend to decrease as graph size increases.

Our computational results lead us to believe that on $G$ :

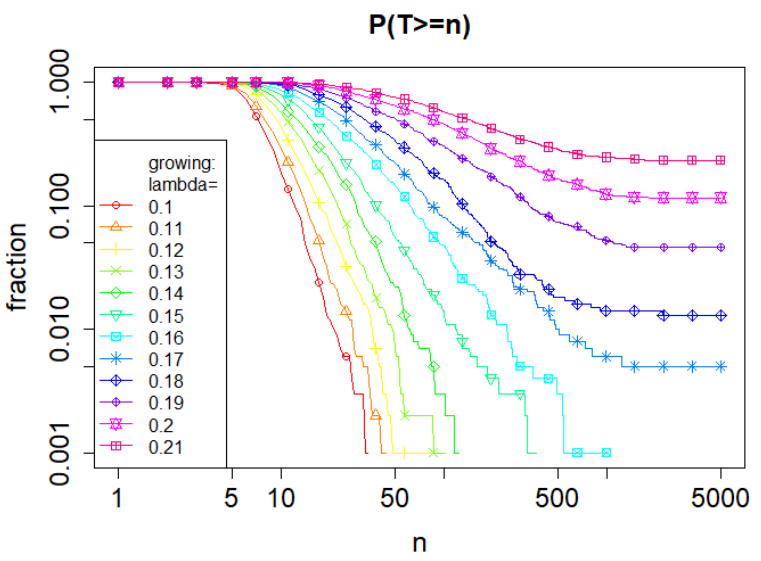

(a)

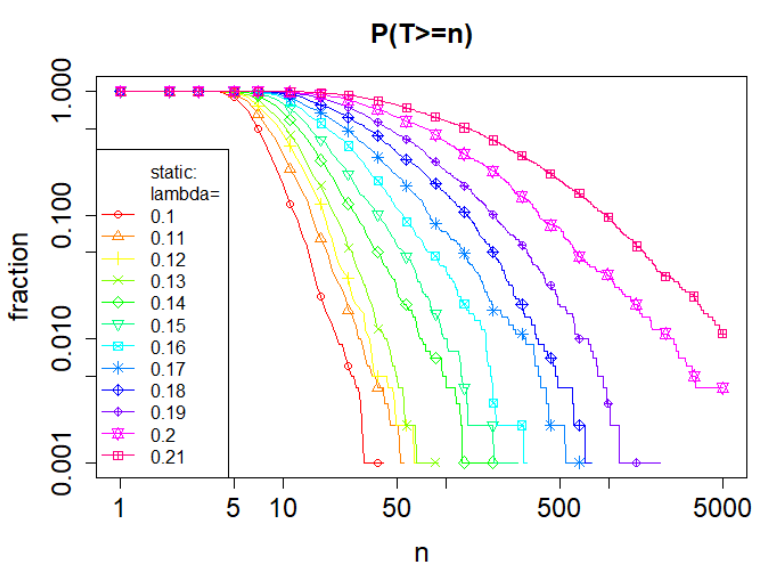

(b)

Fig. 1: Complementary cumulative distribution functions for SIS processes on (a) a growing graph and (b) a static graph. For both graph types, the spreading processes have been run for a range of spreading probabilities $\lambda=$ $0.10,0.11, \ldots, 0.21$, plottedinthisorder, fromlefttorightontheplots. (Markers pictured only for purpose of visual guidance.)

- infinite survival is possible;

- lifetime distribution has a heavier tail compared to that on a static graph;

- the typical notion of epidemic threshold is not as useful to the SIS model on growing graphs.

\section{A. Evidence for infinite survival}

Figure 1 shows the complementary cumulative distribution function (CCDF) of the lifetime of an SIS process on a growing graph (top) and on a static graph (bottom). We notice that the CCDF in the growing case may follow a power law, for at least some values of $\lambda$, and suggests a possibly infinite tail for $\lambda \geq 0.17$.

1) Mass at $\infty$ : One surprising result was seen on the growing graph for large values of $\lambda \geq 0.17$. Once the process had survived for a certain amount of time, it seemed to always survive until termination. Empirically, on simulations 


\section{IEEE/ACM International Conference on Advances in Social Networks Analysis and Mining}

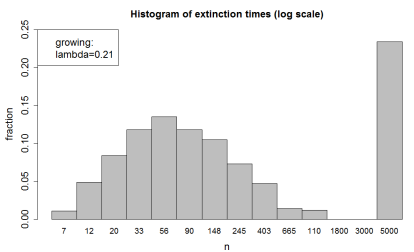

(a)

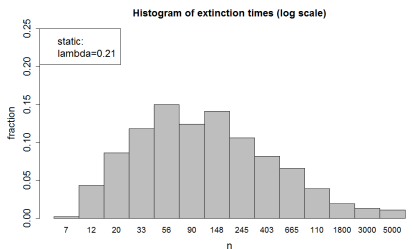

(b)
Fig. 2: Histograms of SIS log-lifetime with $\lambda=0.21$ on (a) growing graph sequences and (b) a static graphs.

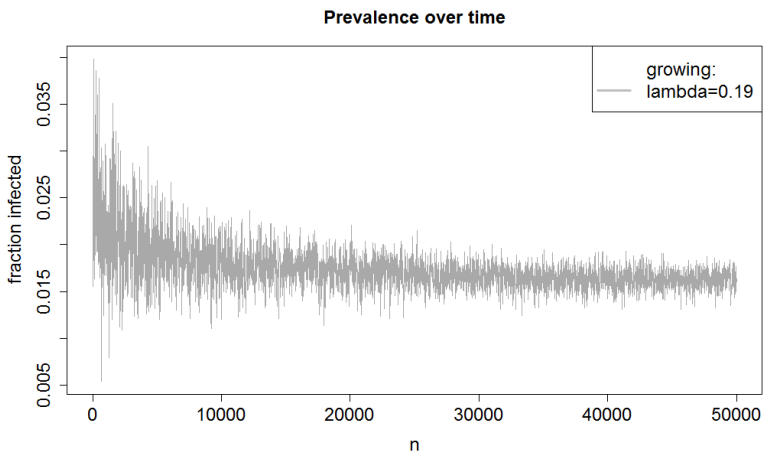

Fig. 3: Fraction of infected nodes at time $n$ for $n=10 k, k=$ $1,2, \ldots, 500$ on a growing graph sequence with $\lambda=0.19$.

terminated at 5000 timesteps, we never observed extinction times in the interval $(2000,5000)$. Letting $\tau$ be the lifetime of our simulated process, there was always a value $\eta \ll 5000$ such that, empirically,

$$
P(\tau \geq 5000 \mid \tau \geq \eta)=1 .
$$

This suggests that if a process survives for a certain number of time steps, then the graph has now grown large enough that future extinction is highly unlikely.

When termination was increased to 10,000 time steps, the same phenomenon occurred, with no extinctions in the interval $(2000,10000)$. We believe this is evidence that such longsurviving processes will survive indefinitely.

On the other hand, on static graphs, we saw extinctions at any point up until termination. Specifically, there were extinctions at around 4000, as seen in the histograms in Figure 2 This shows that in the static case, conditioning on long survival does not guarantee continued survival.

To test the hypothesis of potentially infinite survival in the growing case, we ran one long simulation of 50,000 time steps. The infection prevalence, or the proportion of nodes infected at any time, for this process was recorded every ten time steps and plotted in Figure 3. where the prevalence at time $n$ is defined to be the fraction of infected nodes at time $n$.

We see that the infection prevalence became quite stable over time suggesting that prevalence may converge to this rate as $n \rightarrow \infty$. This indicates a possible steady-state where, once reached, the infection will never become extinct.
2) Conditional survival probability: Letting $\tau$ denote the lifetime of an SIS process, we consider

$$
P(\tau>n+b \mid \tau>n)
$$

the probability that the infection will survive for an additional $b$ timesteps, given that the infection is still present at time $n$. Our main observation for spreading processes on a growing graph is that the conditional survival probability (1) tends to increase as $n \rightarrow \infty$. That is, the longer the infection has survived, the more likely it is to continue to survive.

This quantity is directly related to long-tailed distributions. We say that a random variable $X$ has a long-tailed distribution if for all $t>0$

$$
\lim _{x \rightarrow \infty} P(X>x+t \mid X>x)=1 .
$$

The above statement will be true for any distribution with a mass at infinity, in addition to some non-infinite heavy-tailed distributions.

In our simulations, we fix a value of $\lambda$ and generate 1000 independent graph sequences $G^{(i)}=\left(G_{0}^{(i)}, G_{1}^{(i)}, \ldots\right)$, $i=1, \ldots, 1000$. For each graph sequence $G^{(i)}$, we run the discrete-time SIS model with transmission probability $\lambda$ and record the extinction/termination time $\tau_{i}, i=1, \ldots, 1000$. We estimate (1) for $b=1000$ by plotting

$$
\frac{\sum_{i=1}^{1000} \mathbb{1}\left(\tau_{i}>n+1000\right)}{\sum_{i=1}^{1000} \mathbb{1}\left(\tau_{i}>n\right)}
$$

for $n=1, \ldots, 1000$ in Figure $4 \mathrm{a}$. This process is repeated for several values of $\lambda$.

In Figure 4b, we plot the same estimate for the SIS model on static graphs. Specifically, for each value of $\lambda$, we generate 1000 independent preferential attachment graphs $P A_{n_{0}-1}^{(i)}, i=$ $1, \ldots, 1000$. We run the discrete-time SIS model on each of the static graphs $P A_{n_{0}-1}^{(i)}$, record the extinction/termination time $\tau_{i}^{\prime}$, and plot the fraction resulting from putting $\tau_{i}^{\prime}$ in place of $\tau_{i}$ in (2).

In Figure 4, in both the static and growing cases, we see an increase in the conditional probability as $n$ increases. However, the conditional probability increases faster with $n$ in the growing case than it does in the static case. Further, as $n \rightarrow 5000$, the conditional probability in Figure 4a appears to converge to 1 , whereas in the static case (Figure 4b), the limit seems to be less than 1 . In the growing case, this is evidence of a long-tailed distribution and consistent with mass at infinity.

We must consider, however, an alternate explanation to the increasing slopes in both parts of Figure 4 . Each point in the plots is calculated from the extinction times of the SIS model on 1000 independent graph sequences (or static graphs). It is possible that there are some especially communicable graphs on which an infection is likely survive longer than it would on another graph. For instance, Figure 5 shows two possible outcomes of the preferential attachment model on 50 nodes. The star graph (Figure 5a) is more conducive to spreading than the path (Figure 5b), and thus a large extinction time is more likely on the star graph than the path. 


\section{IEEE/ACM International Conference on Advances in Social Networks Analysis and Mining}

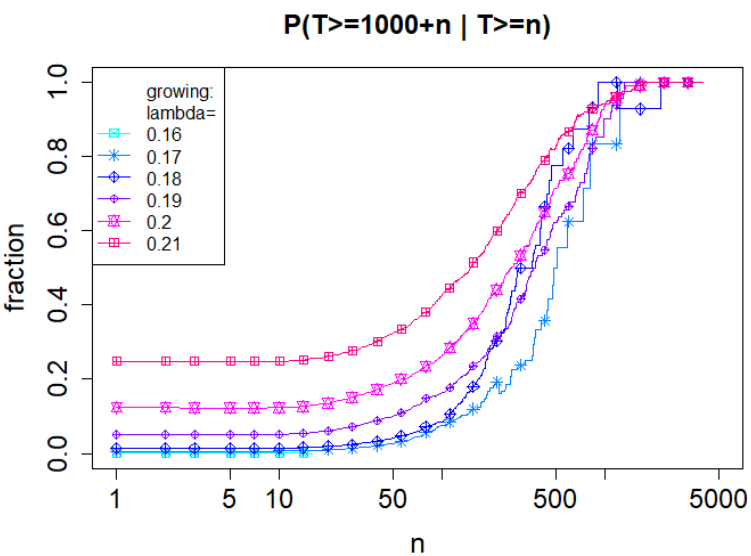

(a)

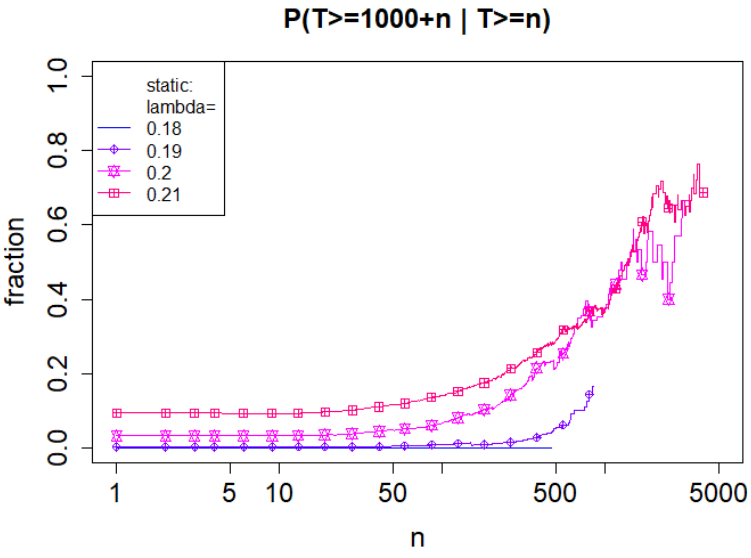

(b)

Fig. 4: Empirical $P(\tau>n+1000 \mid \tau>n)$ for SIS processes on (a) growing graph sequences and (b) a static graphs.

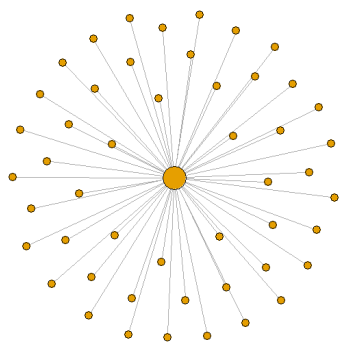

(a)

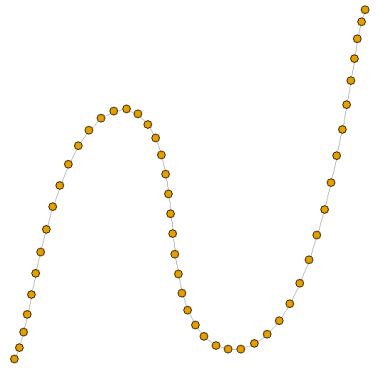

(b)
Fig. 5: (a) Star graph on 50 nodes. (b) Path graph on 50 nodes.
In our analysis we only observe the extinction times, not the graph structure. If we observe a large extinction time, then it is more likely that the underyling graph associated with that extinction time was especially "communicable", and similarly a small extinction time is more likely to have occurred due to a less communicable graph. Therefore, the extinction times $\tau_{i}$ and $\tau_{i}^{\prime}, i=1, \ldots, 1000$ may be positively correlated with some "communicability" property of graph $i$. This correlation may cause a spurious increase with $n$ in the calculation of (2), because if an infection has survived for at least $n$ time steps, then it is more likely to be acting on a "communicable" graph, and therefore more likely to survive for a very long time. Thus, we must make sure that the increase observed in Figure 4 is not only due to this lurking variable.

In short, the increase could be due to the fact that the preferential attachment model may output an entire range of graphs, some of which are more conducive to spreading than others. To understand whether this truly causes a noticeable effect in the plots of Figure 4a, we ran another set of simulations in which 1000 SIS simulations were run on a single graph. That is, we fixed a graph sequence $G=\left(G_{0}, G_{1}, \ldots\right)$, fixed $\lambda=0.17$, and then ran 1000 independent SIS simulations on this single instance of $G$. Letting $\tau_{1}, \ldots, \tau_{1000}$ denote the extinction/termination times, we then plotted

$$
\frac{\sum_{i=1}^{1000} \mathbb{1}\left(\tau_{i}>n+100\right)}{\sum_{i=1}^{1000} \mathbb{1}\left(\tau_{i}>n\right)}
$$

for $n=1, \ldots, 5000$ in Figure 6a This process was repeated 12 times in total, corresponding to the 12 curves in Figure $6 \mathrm{a}$ Note that all curves correspond to $\lambda=0.17$. The difference between the curves correspond to difference graph sequences $G$.

In the static case, we fixed $P A_{n_{0}-1}$, fixed $\lambda=0.19$, and then ran 1000 independent SIS simulations on $P A_{n_{0}-1}$. We plotted the equivalent of (3) for $n=1, \ldots, 5000$ in Figure 6b All curves in this plot correspond to $\lambda=0.19$, but each curve is associated with a different instance of the preferential attachment graph $P A_{n_{0}-1}$.

It should be noted that in Figure 6, we are only looking $b=$ 100 steps into the future, as opposed to $b=1000$ in Figure 4 This is because, for each of the 12 instances of $G$ and $P A_{n_{0}-1}$ in Figure 6 , it was very rare for the infection to survive longer than 1000 time steps, and so taking $b=1000$ does not yield informative results. In comparison, in the data for Figure 4 there were over 1000 instances each of $G$ and $P A_{n_{0}-1}$, and on a few of the SIS simulations, the lifetime was longer than 1000 time steps. This supports our claim of "communicability": that there are some graphs and graph sequences that are especially advantageous for the infection.

In Figure 6b, we see that, for a given static graph, the conditional survival probability $P\left(\tau^{\prime}>n+100 \mid \tau^{\prime}>n\right)$ remains more or less constant with $n$. The curves remain flat and perhaps even decrease as $n$ increases. This is quite different from the previous plot, and indicates that the increasing curves of Figure $4 \mathrm{~b}$ may indeed be spurious, caused solely by 


\section{IEEE/ACM International Conference on Advances in Social Networks Analysis and Mining}

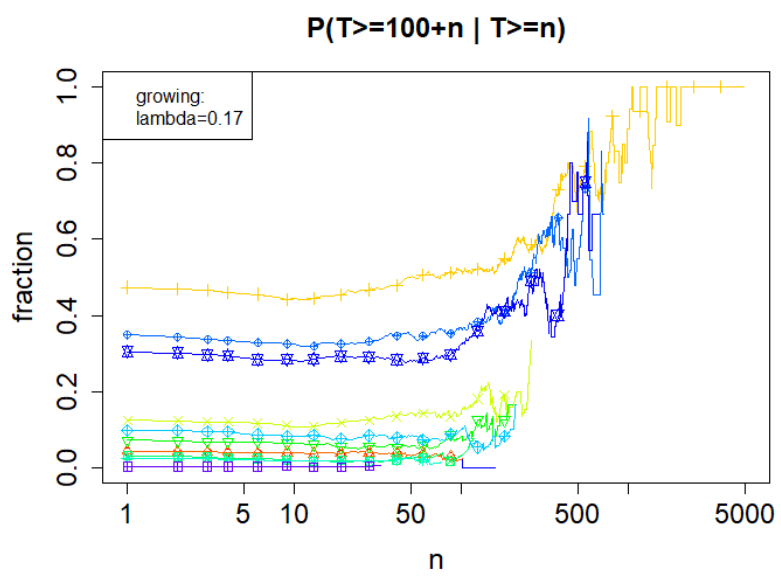

(a)

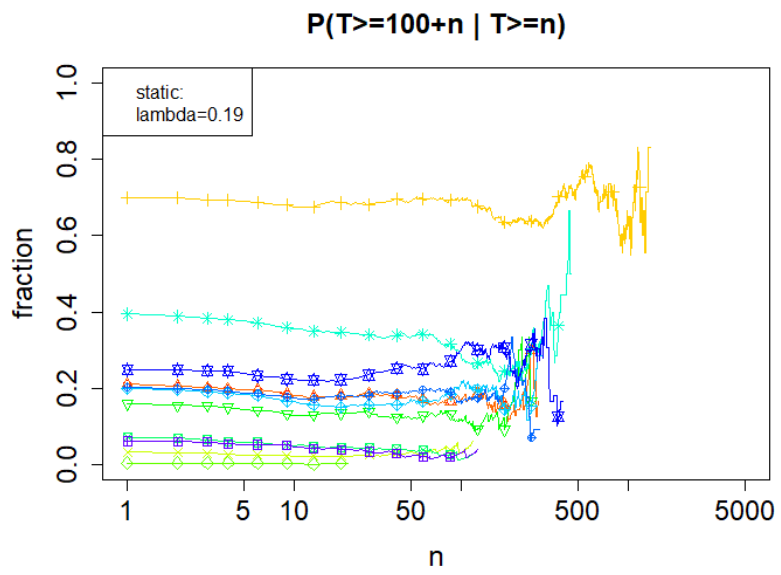

(b)

Fig. 6: Empirical $P(\tau>n+100 \mid \tau>n)$ for SIS processes with (top) $\lambda=0.17$ on a growing graph and (bottom) $\lambda=0.19$ on a static graph. Each curve corresponds to a distinct graph or graph sequence.

differences in the simulated graphs.

For growing graphs, as in Figure 6a, we also see interesting behavior. In many of the simulations, the curve remains flat, while in others, there is an increase as $n$ increases. Therefore we can conclude that for some, but not all, graph sequences, the conditional survival probability $P(\tau>n+100 \mid \tau>n)$ may increase with $n$. In fact, the sharp increase in Figure $4 \mathrm{a}$ could result from an averaging effect over the curves in Figure 6a Therefore, in the growing case, our alternate explanation accounts for some of the increase, but not all. We believe that Figure 6a still shows evidence of long tails, but that the lifetime distribution may be strongly graph-dependent.

In summary, Figure $4 a$ and Figure 6 a show that on growing graphs, the longer an infection has survived, the more likely it is to continue to survive. Further, the lifetime distribution may be long-tailed. Meanwhile, on static graphs, survival up to a certain time does not necessarily imply greater probability of continued survival, as evidenced by Figure $6 \mathrm{~b}$. Figure $4 \mathrm{~b}$ depicts what may be a spurious increase due to a lurking variable: the variation in output of the preferential attachment model. We conclude that for infections on growing graphs, the lifetime distribution may have longer tails, as well as heavier tails, compared to infections on static graphs.

\section{B. Lifetime distributions}

Recall the empirical CCDFs of lifetime in Figure 1. To compare the distributions in the growing and static cases more closely, we consider three regions of $\lambda$. First, consider Figure $7 \mathrm{a}$, where the tails are plotted for both distributions for $\lambda<0.14$.

For these smallest values of $\lambda$, we see virtually no difference between the two cases. This is consistent with the explanation that for $\lambda$ small enough, the process doesn't live long enough to gain the advantage of the growing graph. We conjecture that there is some first critical value $\lambda_{1}$, where if $\lambda<\lambda_{1}$, there is negligible difference between SIS processes on the growing graph and those on the static graph.

Above this critical value, however, there appears to be significant difference in the two cases. In Figure $7 \mathrm{~b}$, for example, the distribution on the growing graph appears to have heavier tails than the distribution on the static graph for $\lambda=0.15,0.16,0.17$. In fact, lifetime in the growing case seems to follow a power law. We conjecture that above the critical value, for $\lambda>\lambda_{1}$, the lifetime in in the growing case has heavier tails than the static case.

In Figure 7c we see the lifetime distributions for $\lambda=$ $0.18,0.19,0.20,0.21$. In this region of $\lambda$, the lifetime distribution appears infinite-tailed for the growing case, but finite for the static case. We conjecture a second critical value, $\lambda_{2} \geq \lambda_{1}$, and that for $\lambda>\lambda_{2}$, SIS processes may survive for an infinite amount of time on growing sequences of graphs.

\section{Epidemic threshold}

Often, we are interested in a critical transmission probability that marks the threshold for "long survival". Whatever the definition of long survival, it is usually the case that the socalled epidemic threshold $\lambda_{c}$ tends to decrease as size of the graph $n$ increases. For this reason, we now argue that these threshold values have minimal relevance to spreading processes on a growing sequence of graphs, because no matter the transmission rate or the size of the initial graph, the process may theoretically survive for long enough that the graph becomes sufficiently large and the relevant threshold becomes sufficiently small.

Suppose that there is some critical $\lambda_{n}$ for each $n$, such that if $\lambda>\lambda_{n}$, then a SIS process on a static graph of size $n$ survives for a long time. Let there be an SIS process with rate $\lambda$ on a sequence of growing graphs $G$ that initializes at $G_{0}$ with size $n_{0}$. If $\lambda \geq \lambda_{n_{0}}$, clearly lifetime will be "long" on the growing graph as well.

On the other hand, suppose $\lambda_{n_{0}+k}<\lambda<\lambda_{n_{0}}$ for some $k$ potentially large. Then long survival is possible as long as the 


\section{IEEE/ACM International Conference on Advances in Social Networks Analysis and Mining}

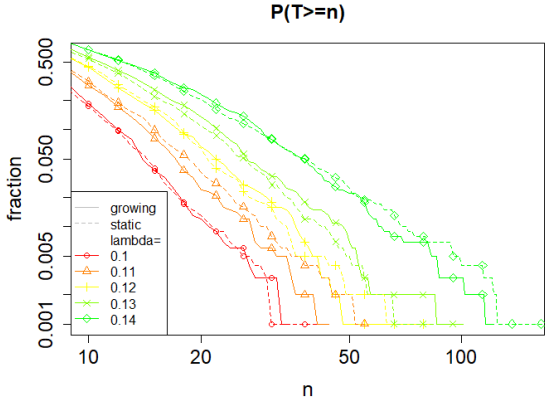

(a) $\lambda$ in the first region.

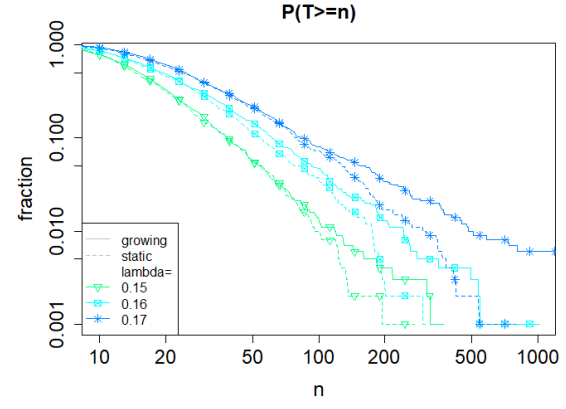

(b) $\lambda$ in the second region.

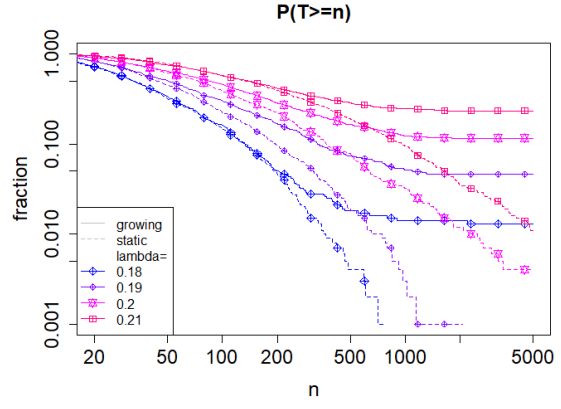

(c) $\lambda$ in the third region.

Fig. 7: Tails of lifetime distributions in the growing (solid) and static (dashed) cases.

virus survives on the growing graph for at least $k$ timesteps. This implies that the critical value $\lambda_{c}$ for $G$ is actually

$$
\lambda_{c}=\liminf _{n \rightarrow \infty} \lambda_{n} .
$$

On preferential attachment networks, the epidemic threshold has been stated to vanish with an increasing number of nodes [2]. In this case, $\liminf _{n \rightarrow \infty} \lambda_{n}=0$, and so the growing preferential attachment graph would theoretically have no epidemic threshold. This means that for any values of $n_{0} \geq 1$ and $\lambda \in(0,1)$, there is a positive probability $p\left(\lambda ; n_{0}\right)$ of long survival. Certainly, $p\left(\lambda ; n_{0}\right)$ may be quite small for small values of $\lambda$, since survival to $k$ timesteps may be highly unlikely. In this case, this notion of epidemic threshold may be of little relevance to practical applications.

For infections on static networks, the epidemic threshold has been defined as the transmission rate above which long survival is possible [4]. In the context of growing networks, however, we posit that it may not be expedient to merely consider the possibility of long survival. Depending on the application, there may be a more useful threshold value, such as a transmission rate corresponding to a critical point in the likelihood of long survival.

\section{CONCLUSION}

We have given evidence that network growth causes a fundamental shift in the distribution of the lifetime of an infection under the SIS model. The addition of new nodes and edges over time may allow the infection to spread further and survive longer than the static case, resulting in a lifetime distribution with heavier tails compared to the static case.

For very small values of the transmission rate parameter $\lambda$, the effect of growth on the lifetime distribution is minimal, as the infection is likely to die out before it has a chance to take advantage of the new connections. However, for larger values of $\lambda$, possibly above some threshold $\lambda_{1}$, the infection may survive for a longer time. If it survives long enough so that the graph has grown sufficiently big, then spreading may become even easier over time. Thus, the longer an infection has survived, the less likely it is to die out in the immediate future. This contributes to heavy-tailed behavior in the lifetime distribution, and possibly long-tailed behavior. For $\lambda$ above a secondary threshold, $\lambda_{2}$, this effect may be strong enough that the infection persists indefinitely.

We also discuss that the epidemic threshold $1 / \rho$, defined for the SIS model on static graphs, may be of minimal utility in the growing case, because the threshold is graph-dependent and decreases as the graph increases.

Future steps would be to prove mathematically the conditions for infinite survival on growing preferential attachment graphs and to study the behavior of infection prevalence as $n \rightarrow \infty$. If infinite survival is possible, then the SIS model on the growing graph may converge to a steady-state, and the steady-state infection prevalence on the growing graph may be related to the steady-state infection prevalence on the infinite graph, which can be difficult to estimate via simulation. If there is a relation between steady-state prevalence on growing and infinite graphs, then the growing graph may be a useful tool to estimate spreading behavior on infinite graphs.

\section{REFERENCES}

[1] Eytan Bakshy, Itamar Rosenn, Cameron Marlow, and Lada Adamic. The role of social networks in information diffusion. In Proceedings of the 21 st International Conference on World Wide Web, WWW '12, pages 519-528, New York, NY, USA, 2012. ACM.

[2] Marc Barthélemy, Alain Barrat, Romualdo Pastor-Satorras, and Alessandro Vespignani. Dynamical patterns of epidemic outbreaks in complex heterogeneous networks. Journal of Theoretical Biology, 235(2):275 $288,2005$.

[3] Güven Demirel, Edmund Barter, and Thilo Gross. Dynamics of epidemic diseases on a growing adaptive network. Scientific Reports, 7:42352, Feb 2017.

[4] A. Ganesh, L. Massoulie, and D. Towsley. The effect of network topology on the spread of epidemics. In Proceedings IEEE 24th Annual Joint Conference of the IEEE Computer and Communications Societies., volume 2, pages 1455-1466 vol. 2, March 2005.

[5] Beniamino Guerra and Jesús Gómez-Gardeñes. Annealed and meanfield formulations of disease dynamics on static and adaptive networks. Phys. Rev. E, 82:035101, Sep 2010.

[6] Adrien Guille, Hakim Hacid, Cecile Favre, and Djamel A. Zighed. Information diffusion in online social networks: A survey. SIGMOD Rec., 42(2):17-28, July 2013.

[7] Petter Holme and Jari Saramäki. Temporal networks. Physics Reports, 519(3):97 - 125, 2012. Temporal Networks.

[8] Matthieu Nadini, Kaiyuan Sun, Enrico Ubaldi, Michele Starnini, Alessandro Rizzo, and Nicola Perra. Epidemic spreading in modular time-varying networks. Scientific Reports, 8(1):2352, dec 2018.

[9] Romualdo Pastor-Satorras, Claudio Castellano, Piet Van Mieghem, and Alessandro Vespignani. Epidemic processes in complex networks. Rev. Mod. Phys., 87:925-979, Aug 2015. 


\section{IEEE/ACM International Conference on Advances in Social Networks Analysis and Mining}

[10] Romualdo Pastor-Satorras and Alessandro Vespignani. Epidemic spreading in scale-free networks. Phys. Rev. Lett., 86:3200-3203, Apr 2001.

[11] Péter L. Simon and Istvan Z. Kiss. From exact stochastic to meanfield ODE models: a new approach to prove convergence results. IMA Journal of Applied Mathematics, pages 1-21, 2005.

[12] Eugenio Valdano, Michele Re Fiorentin, Chiara Poletto, and Vittoria Colizza. Epidemic threshold in continuous-time evolving networks. Phys. Rev. Lett., 120:068302, Feb 2018.

[13] Jiyoung Woo and Hsinchun Chen. Epidemic model for information diffusion in web forums: experiments in marketing exchange and political dialog. SpringerPlus, 5(1):66, Jan 2016. 